\title{
Cuidador primario, agente transmisor de infecciones asociadas a la atención de salud: revisión de literatura
}

\author{
Blancarte-Fuentes, Enrique ${ }^{i *}$; Álvarez-Aguirre, Alicia²; Tolentino-Ferrel, María del Rosario ${ }^{3}$
}

\begin{abstract}
RESUMEN
Introducción: Las Infecciones Asociadas a la Atención en Salud representan un problema de salud pública, aumentan los costos en la atención médica, la morbilidad y mortalidad, existen factores de riesgo ya identificados, entre ellos los humanos, sin embargo "el cuidador" se ha estudiado poco, siendo este un reservorio que pudiera ser el principal agente transmisor de una o varias infecciones. Objetivo: indagar en la evidencia científica actual relacionada a infecciones comunes, factores de riesgo, los conocimientos, prácticas y aptitudes del cuidador y su relación con las Infecciones Asociadas a la Atención en Salud. Metodología: Revisión sistemática de la literatura, realizada en base de datos electrónicas: PubMed, BVS, Trip Database, Science Direct, Ebsco Host y Google académico; se analizaron documentos publicados de 2013 a 2018, en idioma inglés, portugués y español, se usaron los términos MESH y DeCs "infección nosocomial", "epidemiología", "cuidadores" y "atención al paciente", se plantea una pregunta PICO, los estudios seleccionados incluyeron metaanálisis, ensayos clínicos controlados aleatorios, revisiones sistemáticas y estudios cualitativos, los criterios de exclusión fueron estudios con cuidadores en domicilios y en unidades de cuidados intensivos adultos y pediátricos. Resultados y conclusiones: se obtuvieron 419 artículos, 15 que cumplieron con los criterios de inclusión los cuales muestran los tipos de infecciones más comunes, factores de riesgo, conocimientos, prácticas y aptitudes del cuidador en la prevención de IAAS, concluyendo que existe poca evidencia científica, deficiencias del conocimiento, programas e intervenciones dirigidas al cuidador para la prevención de las IAAS.
\end{abstract}

Palabras clave: Infección hospitalaria; cuidadores; atención al paciente (DeCS).

\footnotetext{
${ }_{1}^{1}$ Universidad de Guanajuato Celaya, Gto., México. Correo Electrónico: e.blancarte@ugto.mx. ORCID-ID: https://orcid.org/0000-0001-5768-2899 2 Universidad de Guanajuato Celaya, Gto., México. Correo Electrónico: alicia.alvarez@ugto.mx. ORCID-ID: https://orcid.org/0000-0001-5538-7634 ${ }_{3}^{3}$ Universidad de Guanajuato Celaya, Gto., México. Correo Electrónico: maria.tolentino@ugto.mx. ORCID-ID: https://orcid.org/0000-0002-46905564
}

Recibido: 30/05/2019 Aceptado: $12 / 12 / 2019$

*Autor para correspondencia

\section{Cómo citar este artículo}

Blancarte-Fuentes E, Álvarez-Aguirre A, Tolentino-Ferrel MR. Cuidador primario, agente transmisor de infecciones asociadas a la atención de salud: revisión de literatura. SANUS. 2019; (12): 34-50. [Acceso___ _ ]; Disponible en:

$$
\text { mes día año URL }
$$




\title{
Cuidador primário, agente transmissor de infecções associadas a cuidados de saúde: revisão de literatura
}

\begin{abstract}
ABSTRATO
Introdução: As infecções associadas aos cuidados de saúde representam um problema de saúde pública, aumentam os custos com cuidados médicos, morbidade e mortalidade. Os seres humanos já estão identificados como fatores e risco; no entanto, "o cuidador" mal foi estudado, e ele pode ser um reservatório e transmissor de uma ou várias infecções. Objetivo: investigar as evidências científicas atuais relacionadas a infecções comuns, fatores de risco, conhecimento, prática e aptidão do cuidador e sua relação com infecções associadas à assistência á saúde. Métodos: Revisão sistemática da literatura realizada com base em dados electrônicos, nomeadamente, PubMed, BVS, Trip Database, Science Direct, Ebsco Host, e Google Scholar. Os documentos publicados de 2013 a 2018, em inglês, português e espanhol, foram avaliados; foram utilizados os termos MESH e DeCs, "infecção hospital", "epidemiologia", "cuidadores", e "assistência ao paciente". Uma questão do PICO foi levantada, os estudos selecionados incluíram metanálise, ensaios clínicos randomizados e controlados, revisões sistemáticas e estudos qualitativos. Os critérios de exclusão foram compostos por estudos que incluíram cuidadores em casa e em unidades de terapia intensiva, tanto para adultos quanto para crianças. Resultados e Conclusões: : Foram obtidos 419 artigos; 15 deles atenderam aos critérios de inclusão, que mostrou o tipo de infecções mais comuns, bem como fatores de risco, conhecimentos, práticas e aptidões dos cuidadores na prevenção do IAAS (infecções associadas a cuidados de saúde) (por sua sigla em espanhol), concluindo que há pouca evidencia científica, e deficiências no conhecimento, programas e intervenções dirigidas ao cuidador para prevenir o
\end{abstract} IAAS.

Palavras chave: Infecção nosocomial; cuidador; cuidados para o paciente (DeCS).

\section{INTRODUCCIÓN}

Una IAAS, también denominadas infecciones nosocomiales u hospitalarias, son aquellas que el paciente puede contraer al recibir cuidados de salud durante su estancia en un hospital, ampliándose a cuidados ambulatorios, hospitalización en domicilio, o instituciones geriátricas (1).

La aparición de una IAAS sigue siendo la complicación más frecuente en pacientes hospitalizados, la magnitud y el impacto implica un incremento en la morbilidad y mortalidad, la prolongación de la estancia hospitalaria, discapacidad permanente, aumento de la resistencia a antimicrobianos y elevado costo económico y social para los sistemas sanitarios (2,3), además, se consideran como el primer evento adverso de mayor frecuencia en las unidades de cuidados intensivos y representan entre 20 y $30 \%$ de las complicaciones graves evitables (4).

La carga general de las IAAS sigue siendo alta, la Organización Mundial de la Salud (OMS) identificó que
$7 \%$ de los pacientes en los países desarrollados y 16\% en los países en desarrollo tienen al menos una infección en cualquier momento durante la hospitalización, con una tasa de mortalidad estimada de 10\% (5).

La incidencia y prevalencia más comunes son; infección de tracto urinario asociado al uso de catéter, neumonía asociada al uso de ventilador, infección del sitio quirúrgico e infección del torrente sanguíneo asociado al uso de catéter, las cuales pueden evolucionar de forma peligrosa y a veces potencialmente mortales, este tipo de infección constituyen aproximadamente $80 \%$ de todas las infecciones ${ }^{(6-8)}$.

Cada año en Estados Unidos de América, los Centros para la Prevención y Control de Enfermedades (CDC), estiman que hay 1,7 millones de casos, lo que contribuye a 98,000 mil muertes y una estimación de costos en la atención de \$10 mil millones de dólares ${ }^{(9)}$.

En Europa la prevalencia, principalmente en Alemania fue de $5.1 \%$ en 2011, involucrando aproximadamente $8,000.00$ pacientes anualmente, otra encuesta de prevalencia aplicada 
a 231,459 pacientes de 947 hospitales de países europeos en el año 2011 reveló que en un momento dado 5.7\% de los pacientes adquirió una infección ${ }^{(10)}$.

La bacteriemia hospitalaria en varios países europeos afectó a uno de cada 20 pacientes hospitalizados, lo que correspondió a un total, anual, de 4,1 millones de pacientes; de estos, se estima que 37.000 pacientes fallecen cada año (11).

En un estudio, de prevalencia de IAAS realizado en México durante el 2011 a instancias de la Secretaría de Salud, se reportó una prevalencia puntual de $21 \%$, lo cual es prácticamente el doble de los estándares internacionales que se encuentran entre el 5 y $10 \%{ }^{(13)}$. Otro estudio estimo una tasa de 3.8 a 26 por cada 100 egresos hospitalarios (12).

En el estado de Guanajuato en promedio cada día, más de cuatro personas contraen una infección cuando reciben tratamiento en algún centro de salud u hospital, en el 2018 se reportaron 1602 casos, de los cuales 853 se presentaron en varones y 749 en mujeres, identificando como causas: la falta de higiene en el instrumental médico o las instalaciones, el propio padecimiento del paciente, y falta de medidas de higiene del cuidador y visitantes ${ }^{(13)}$.

Las instituciones que otorgan servicios de salud requieren la presencia de un cuidador que acompaña a la persona enferma, su intervención es insustituible por los lazos de parentesco que existen entre ambos, ya que el cuidador asume la responsabilidad de atender las necesidades básicas, toma decisiones y se convierte en el responsable del enfermo $(14,15)$

La aparición de una IAAS resulta de la interacción entre un agente infeccioso y un huésped susceptible, en esta interacción existe un elemento denominado cadena de infección conformada por: agente infeccioso, reservorio, puerta de salida, modo de transmisión, puerta de entrada y huésped susceptible, aquí es donde se identifica al cuidador como un reservorio y agente de transmisión de una infección por formar parte del ambiente hospitalario ${ }^{(6)}$.

Múltiples estudios se han enfocado a investigar cada uno de los elementos de la cadena de infección que ocasionan una IAAS, pero poco se ha estudiado sobre la participación del cuidador como factor de prevención y potencial reservorio, capaz de albergar o portar algún agente que pueda transmitir a la persona enferma durante el cuidado, a pesar de que el cuidador en el hospital realiza múltiples actividades que van desde el acompañamiento emocional hasta el apoyo en el aseo, la medicación y la manipulación de dispositivos invasivos y no invasivos (17).

Cuidar es según la Real Academia Española: "un acto individual que uno se da a sí mismo cuando adquiere autonomía", pero también es un acto de reciprocidad que se tiende a dar a cualquier persona que, temporal o definitivamente, requiere ayuda para asumir necesidades vitales (20).

El cuidador es "aquella persona no profesional que de forma cotidiana se hace cargo de las necesidades básicas y psicosociales del enfermo o lo supervisa en su vida diaria", reside en su gran mayoría en el mismo domicilio, es quien está siempre disponible para satisfacer sus demandas y no recibe retribución económica alguna por la función que desempeña ${ }^{(14,15)}$

Diferentes estudios sociológicos dejan patente la cifra que representa en la actualidad los cuidadores informales. En la población norteamericana se ha calculado que 19,2\% (52 millones de personas) cuida a personas adultas enfermas. En Canadá, 9,6\% de la población (3 millones de personas) presta ayuda a individuos afectados por alguna enfermedad crónica ${ }^{(18)}$.

La Encuesta Nacional de Ocupación y Empleo (ENOE), al tercer trimestre de 2016 registró 286 mil cuidadores de personas dependientes en México, los datos revelaron que este rol lo asumen principalmente las mujeres ya que de cada 100 cuidadores 97 son del sexo femenino, además de cada 100 de ellos, 38 se desempeñan en establecimientos de salud y 62 en viviendas particulares. En promedio, laboran 38.5 horas a la semana y ganan 24.3 pesos por hora trabajada ${ }^{(19)}$.

El objetivo fue realizar una revisión sistemática en la evidencia científica existente que revele las conductas del cuidador y su relación con las IAAS, con el propósito de identificar como es que el cuidador puede ser causante de una infección, cuando está colaborando en el cuidado.

\section{METODOLOGÍA}

Este estudio fue una revisión sistemática siguiendo los elementos de la declaración PRISMA, se realizo busquedas en bases de datos electrónicas como PubMed, BVS, Trip Database, Science Direct, Ebsco Host y Google Académico, publicados de 2013 a 2018, se eligieron por su impacto en el área de salud.

Se utilizaron las siguientes estrategias de búsqueda: la primera búsqueda en PubMed con el siguiente algoritmo de busqueda: ("Cross Infection/diagnosis"[Mesh] OR "Cross Infection/epidemiology" [Mesh] OR "Cross Infection/etiology" OR "Cross Infection/organization and administration"[Mesh] OR "Cross Infection/transmission"[Mesh]) AND ((Clinical Trial[ptyp] OR Review[ptyp] OR Meta-Analysis[ptyp] OR Practice Guideline[ptyp] OR systematic[sb]) AND ("loattrfree full text"[sb] AND "loattrfull text" [sb]) AND "2013/04/17"[PDat]: "2018/04/15"[PDat] AND "humans"[MeSH Terms] AND (English[lang] OR Portuguese[lang] OR Spanish[lang]) AND ("female"[MeSH Terms] OR "male"[MeSH Terms]) AND ("adult"[MeSH Terms] OR "adult"[MeSH Terms:noexp] OR "aged"[MeSH Terms])); la segunda busqueda en Biblioteca Virtual en Salud con terminos DeCs y operadores boleanos: "cross infection" AND caregiver; la tercera busqueda con terminus DeCs y operadores boleanosTrip Database "cross infection" AND caregiver; y Science direct: "nosocomial infection" AND caregiver, adicionalmente en google académico con terminos DeCs.

Los criterios de inclusión fueron: estudios de ensayos clínicos 
aleatorios, revisiones sistemáticas, guías de práctica clínica y estudios cualitativos publicados en inglés, portugués y español que abordaron las infecciones hospitalarias y su relación con el cuidador de pacientes adultos en hospitales. Los criterios de exclusión fueron cuidadores de pacientes en domicilios y cuidadores en unidades de cuidados intensivos adultos y pediátricos.

La identificación y selección de artículos fueron realizados de forma independiente por 2 revisores (EBF y MRTF), el analisís se realizó por un tercer revisor (AAA), los artículos se almacenaron y los duplicados identificados en varías bases de datos se almacenaron en Mendeley, versión 19.5.

\section{RESULTADOS}

De los 419 artículos encontrados en la búsqueda de la literatura: 92 documentos se localizaron en PubMed; 29 en Biblioteca Virtual en Salud; 52 Trip Database, en Science Direct 233 artículos, en EbscoHost 13 y en otras fuentes consultadas como Google Académico 10 artículos; después de realizar la lectura crítica de los documentos, se aceptaron un total de 15 artículos (Fig. 1).

El tipo de estudio de los artículos seleccionados fue: revisión sistemática (5), descriptivo/observacional (6), cuasiexperimental (2), cualitativo (1), cartas al editor (1). El idioma en 14 artículos fue inglés, sólo uno en idioma español, el orígen del artículo fue en Colombía (1), Haití y Etiopía (1), Bangladesh (3), Estados Unidos de América (10), el año de artículo varia de 2013 a 2018, lo cual se resume en la tabla 1. Esta revisión tuvo el objetivo de realizar una revisión sistemática en la evidencia científica existente que evidenciara las conductas del cuidador y su relación con las IAAS, tras el análisis de los documentos se rescataron tres aspectos relevantes: a) Tipos de infecciones hospitalarias; b) Factores de riesgo de una IAAS y c) Prácticas, conocimientos y actitudes de los cuidadores para prevenir una IAAS.

\section{Tipos de infecciones hospitalarias}

Los tipos de infecciones más frecuentes incluyeron las infecciones del torrente sanguíneo asociados con la línea central, infecciones del tracto urinario asociadas con catéter urinario, infecciones en el sitio quirúrgico y neumonía asociada con el ventilador (21).

Figura 1. Diagrama de flujo que resume el proceso para la identificación de estudios elegibles.

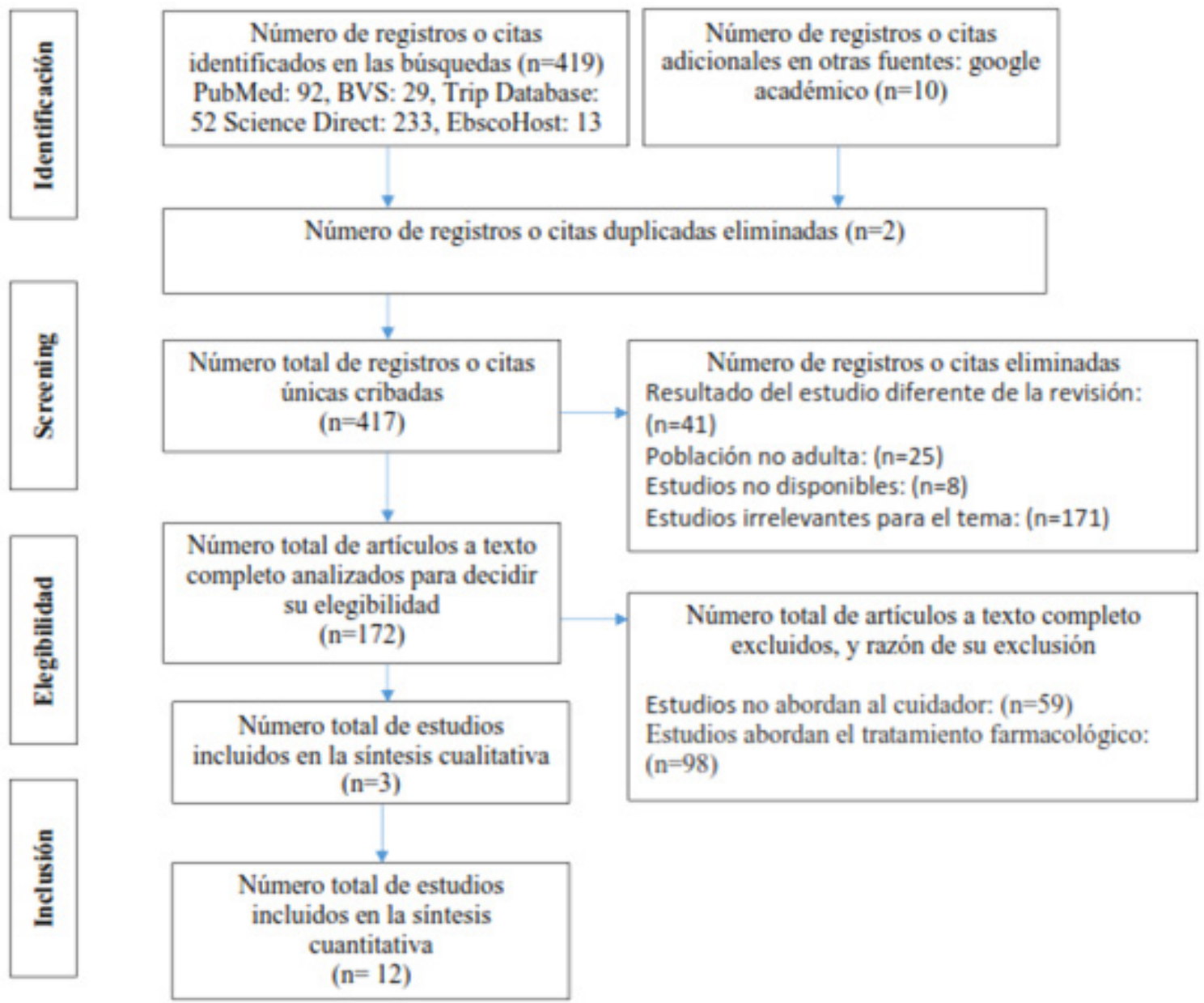

Fuente: Elaboración propia 
Tabla 1. Descripción de artículos seleccionados en esta revisión sistemática

\begin{tabular}{|c|c|c|c|}
\hline Titulo, autores y país & Revista y año & Tipo de estudio & Metodología \\
\hline $\begin{array}{l}\text { Factores de riesgo de infec- } \\
\text { ción asociada a la atención } \\
\text { en salud en adultos hospita- } \\
\text { lizados: revisión sistemática } \\
\text { y metaanálisis }\end{array}$ & \begin{tabular}{|l|} 
American Journal of \\
Infection Control 2017
\end{tabular} & $\begin{array}{l}\text { Revisión sistemática y } \\
\text { metaanálisis }\end{array}$ & $\begin{array}{l}\text { Búsqueda en base de datos electró- } \\
\text { nicas: MEDLINE, Embase y LILACS, se } \\
\text { incluyeron estudios de 2009-2016, se } \\
\text { obtuvieron } 867 \text { estudios, } 65 \text { cumplie- } \\
\text { ron con los criterios de revisión y se } \\
\text { utilizaron } 18 .\end{array}$ \\
\hline $\begin{array}{l}\text { Prácticas basadas en la } \\
\text { evidencia para aumentar el } \\
\text { cumplimiento de la higie- } \\
\text { ne de manos en centros } \\
\text { de atención en salud: una } \\
\text { revisión integrada }\end{array}$ & $\begin{array}{l}\text { American Journal of } \\
\text { Infection Control } 2017\end{array}$ & Revisión sistemática & $\begin{array}{l}\text { Búsqueda en base de datos electróni- } \\
\text { cas: MEDLINE, se incluyeron estudios } \\
\text { de } 2002-2015 \text {, se obtuvieron } 151 \text { estu- } \\
\text { dios, } 73 \text { cumplieron con los criterios de } \\
\text { revisión. }\end{array}$ \\
\hline $\begin{array}{l}\text { Infecciones del torrente } \\
\text { sanguíneo en pacientes } \\
\text { mayores }\end{array}$ & La virulencia 2016 & Revisión sistemática & $\begin{array}{l}\text { Búsqueda en base de datos electróni- } \\
\text { cas: Pubmed hasta septiembre de } 2015\end{array}$ \\
\hline $\begin{array}{l}\text { Factores de riesgo para } \\
\text { bacteriemia nosocomial } \\
\text { secundaria a bacteriuria } \\
\text { asociada a catéter urinario: } \\
\text { una revisión sistemática }\end{array}$ & Urol Nurse 2015 & Revisión sistemática & $\begin{array}{l}\text { Búsqueda en base de datos electróni- } \\
\text { cas: MEDLINE, Scopus, Cochrane, Cl- } \\
\text { NAHL y la base de datos de brotes con } \\
\text { la ayuda de un bibliotecario médico, se } \\
\text { incluyeron estudios de 1983-2012, se } \\
\text { obtuvieron } 5231 \text { estudios, } 79 \text { cumplie- } \\
\text { ron con los criterios de revisión y se } \\
\text { utilizaron } 7 \text {. }\end{array}$ \\
\hline $\begin{array}{l}\text { Un sistema automatizado } \\
\text { de cumplimiento de higiene } \\
\text { de manos está asociado con } \\
\text { un mejor monitoreo de la } \\
\text { higiene de manos }\end{array}$ & $\begin{array}{l}\text { American Journal of } \\
\text { Infection Control } 2017\end{array}$ & Cuasi/experimental & $\begin{array}{l}\text { Cohorte retrospectivo implementando } \\
\text { un sistema de control automatizado de } \\
\text { cumplimiento de higiene de manos }\end{array}$ \\
\hline $\begin{array}{l}\text { El aumento en el rendi- } \\
\text { miento de la higiene de las } \\
\text { manos disminuye los marca- } \\
\text { dores de infecciones noso- } \\
\text { comiales y su costo después } \\
\text { de la implementación de un } \\
\text { sistema automatizado de } \\
\text { monitoreo de higiene de } \\
\text { manos }\end{array}$ & $\begin{array}{l}\text { American Journal of } \\
\text { Infection Control } 2014\end{array}$ & Cuasi/experimental & $\begin{array}{l}\text { Instalación de un sistema inalámbrico } \\
\text { de monitoreo de higiene de manos, } \\
\text { monitoreando los dispensadores y eva- } \\
\text { luaron a médicos, enfermeras, pacien- } \\
\text { tes, cuidadores y otros profesionales de } \\
\text { la salud. }\end{array}$ \\
\hline $\begin{array}{l}\text { Cuidadores familiares en } \\
\text { hospitales públicos tercia- } \\
\text { rios en Bangladesh: riesgos } \\
\text { y oportunidades para el } \\
\text { control de infecciones } \\
\end{array}$ & $\begin{array}{l}\text { American Journal of } \\
\text { Infection Control } 2014\end{array}$ & Cualitativo & $\begin{array}{l}\text { Se realizo una observación de } 48 \text { horas } \\
\text { en } 3 \text { hospitales públicos y se realizaron } \\
12 \text { entrevistas a cuidadores familiares }\end{array}$ \\
\hline $\begin{array}{l}\text { Infecciones del torrente san- } \\
\text { guíneo en medicina interna }\end{array}$ & La virulencia 2016 & Revisión sistemática & $\begin{array}{l}\text { búsqueda en base de datos electróni- } \\
\text { cas: MEDLINE, PubMed }\end{array}$ \\
\hline $\begin{array}{l}\text { Comentario sobre: Cuidado- } \\
\text { res familiares en hospitales } \\
\text { públicos de atención tercia- } \\
\text { ria en Bangladesh: riesgos } \\
\text { y oportunidades para el } \\
\text { control de infecciones }\end{array}$ & $\begin{array}{l}\text { American Journal of } \\
\text { Infection Control } 2014\end{array}$ & Cartas al editor & Comentario al artículo publicado \\
\hline
\end{tabular}




\begin{tabular}{|c|c|c|c|}
\hline $\begin{array}{l}\text { Higiene de las manos del } \\
\text { personal sanitario y de los } \\
\text { cuidadores familiares en las } \\
\text { instalaciones sanitarias de } \\
\text { Bangladesh: resultados de } \\
\text { la encuesta de referencia de } \\
\text { higiene nacional de Bangla- } \\
\text { desh }\end{array}$ & $\begin{array}{l}\text { The Journal of Hospi- } \\
\text { tal Infection } 2016\end{array}$ & $\begin{array}{l}\text { Descriptivo/observa- } \\
\text { cional }\end{array}$ & $\begin{array}{l}\text { Se utilizo la encuesta de referencia } \\
\text { de higiene de manos, se aplicó a } 100 \\
\text { grupos de población rural y urbana, se } \\
\text { examinó la infraestructura de higiene } \\
\text { de manos en } 875 \text { centros hospitalarios } \\
\text { y se observó el comportamiento en } \\
100 \text { centros }\end{array}$ \\
\hline $\begin{array}{l}\text { ¿Qué saben los visitantes y } \\
\text { cómo se sienten acerca de } \\
\text { las precauciones de con- } \\
\text { tacto? }\end{array}$ & $\begin{array}{l}\text { The Journal of Hospi- } \\
\text { tal Infection } 2018\end{array}$ & $\begin{array}{l}\text { Descriptivo/observa- } \\
\text { cional }\end{array}$ & $\begin{array}{l}\text { Se aplico la encuesta utilizando el } \\
\text { marco conceptual de la Iniciativa de In- } \\
\text { geniería de Sistemas para la Seguridad } \\
\text { del Paciente a visitantes de pacientes } \\
\text { hospitalizados al azar }\end{array}$ \\
\hline $\begin{array}{l}\text { Conocimientos y actitudes } \\
\text { de los visitantes a pacientes } \\
\text { en aislamiento de contacto }\end{array}$ & $\begin{array}{l}\text { American Journal of } \\
\text { Infection Control } 2014\end{array}$ & $\begin{array}{l}\text { Descriptivo/observa- } \\
\text { cional }\end{array}$ & $\begin{array}{l}\text { Se aplico la encuesta en unidades hos- } \\
\text { pitalarias a } 137 \text { pacientes en aislamien- } \\
\text { to de contacto }\end{array}$ \\
\hline $\begin{array}{l}\text { Evaluación de la com- } \\
\text { prensión y satisfacción del } \\
\text { paciente y del cuidador con } \\
\text { el uso de aislamiento de } \\
\text { contacto }\end{array}$ & $\begin{array}{l}\text { American Journal of } \\
\text { Infection Control } 2016\end{array}$ & $\begin{array}{l}\text { Descriptivo/observa- } \\
\text { cional }\end{array}$ & $\begin{array}{l}\text { Se aplico la encuesta en unidades hos- } \\
\text { pitalarias a } 500 \text { pacientes en aislamien- } \\
\text { to de contacto }\end{array}$ \\
\hline $\begin{array}{l}\text { Identificación de los deter- } \\
\text { minantes psicológicos del } \\
\text { lavado de manos: resultados } \\
\text { de dos estudios de cuestio- } \\
\text { nario de corte transversal en } \\
\text { Haití y Etiopía }\end{array}$ & $\begin{array}{l}\text { American Journal of } \\
\text { Infection Control } 2015\end{array}$ & $\begin{array}{l}\text { Descriptivo/observa- } \\
\text { cional }\end{array}$ & $\begin{array}{l}\text { Se utilizaron entrevistas personales a } \\
1274 \text { cuidadores principales }\end{array}$ \\
\hline $\begin{array}{l}\text { Factores psicosociales en } \\
\text { cuidadores y riesgo de } \\
\text { infección del torrente circu- } \\
\text { latorio en pacientes en una } \\
\text { institución de Medellín, } 2014\end{array}$ & $\begin{array}{l}\text { CES Salud Pública } \\
2015\end{array}$ & $\begin{array}{l}\text { Descriptivo/observa- } \\
\text { cional }\end{array}$ & $\begin{array}{l}\text { Se siguió a } 177 \text { cuidadores, además } \\
\text { que el se analizó la atención en } 56 \text { pa- } \\
\text { cientes a quienes se les coloco catéter }\end{array}$ \\
\hline
\end{tabular}

Fuente: Elaboración propia

\section{Factores de riesgo de una IAAS}

Si bien no existe un consenso sobre los riesgos, se identificaron como factores extrínsecos para adquirir una IAAS, la colocación y manipulación de catéter venoso central, tiempo de hospitalización, cateterización y manipulación de la sonda vesical, aspiración de secreciones, sonda de gastrostomía y falta de aplicación de técnicas de medidas específicas ${ }^{(22)}$.

Como determinantes de las IAAS es que no se ha estudiado a profundidad que pasa desde la inserción de un dispositivo hasta la aparición de una infección (23).

Otros factores son: el desconocimiento por el uso inadecuado de las técnicas, escaso conocimiento de las medidas básicas de control de infecciones, falta de políticas de control, la pobreza, la falta de personal para el cuidado de la salud supliendo esta falta por el cuidador, la transmisión de patógenos durante el contacto directo con los pacientes y el ambiente ya que un paciente puede ser un reservorio más para el paciente no infectado (21).

Otros factores de riesgo son: postración en cama, presencia de acceso intravascular o gastrostomía en la admisión, institucionalización, exposición a instrumentos y procedimientos, así como incontinencia urinaria (24), manipulación de dispositivos permanentes de forma simultánea del catéter venoso central y catéter urinario se asocian de manera importante en la aparición de una infección del torrente sanguíneo ${ }^{(25)}$.

El entorno familiar, social, económico, el estrés y grado de escolaridad del cuidador representan un riesgo muy alto para que sean factores desencadenantes de una IAAS (26). Las dificultades para el acceso a materiales como jabón y lugares para el lavado, la mala higiene ambiental en el entorno del enfermo, condiciones no adecuadas de las 
instalaciones, y la falta de capacitación en control de infecciones son factores que predisponen a la transmisión de infecciones ${ }^{(27)}$.

Otro factor encontrado fue que el cumplimiento de las precauciones por contacto por el personal de salud afecta en gran medida el cumplimiento del visitante, lo que se asocia a una percepción de bajo riesgo para no realizar las precauciones de contacto (28).

No existe un método estandarizado de educación dirigida a cuidadores primarios y visitantes a pesar de que los visitantes parecen comprender el propósito de las medidas de precaución en el entorno de pacientes hospitalizados, además de que prevalece el desconocimiento del cuidador primario sobre la presencia de una $\mathrm{IH}$ en el paciente ${ }^{(29)}$.

La exposición repetida y continua, una higiene de manos inadecuada y falta de conocimientos sobre la transmisión de las infecciones, ponen de relieve al cuidador como factor de riesgo y a la vez determinante para la transmisión de una IAAS (30).

\section{Genero, escolaridad, prácticas, conocimientos y actitudes de los cuidadores para prevenir una IH}

Referente al sexo se identifico que el cuidador entre el 60 y $70 \%$ es mujer y con nivel mínimo de educación secundaria y el resto educación universitaria (29), en relación al conocimiento sobre el aislamiento de pacientes, los cuidadores comprenden que es una medida preventiva y de protección contra una IAAS, en cuanto al uso de equipo de protección personal es bajo ${ }^{(28,31,32)}$. Continuando con la falta de conocimiento se asocia a la motivación lo que se refleja en el comportamiento del cuidador familiar de no seguir las normas de prevención (27).

En cuanto a la atención el cuidador familiar brindo atención las 24 horas realizando actividades de limpieza y apoyo psicológico, en total proporcionaron 2065 tareas de cuidado, el $75 \%(1,544)$ involucro contacto directo con el paciente, se observo que solo en 4 ocasiones se lavaron las manos con jabón (33).

Las políticas de aislamiento generan seguridad, satisfacción y respuesta positiva, pero disminuye el tiempo de contacto y examen directo de los trabajadores de la salud lo que impacta en la calidad de la atención, además de que genera mayores eventos adversos, depresión y delirio del paciente, en este contexto se pone de relieve la generación y exploración de estudios que investiguen a fondo la atención que brindan, además de generar estrategias para mejorar el conocimiento de los cuidadores sobre la transmisión y la reducción a la exposición a los agentes causales ${ }^{(32)}$.

Una revisión integrada sugiere cinco categorías de intervención para prevenir una $\mathrm{IH}$ : (1) mejorar la conciencia con la educación (transferencia de conocimiento, evaluación tutoría y retroalimentación), (2) diseño y planificación de las instalaciones, (3) protocolos y procedimientos a nivel individual, (4) programas a nivel institucional y (5) intervenciones multimodales que involucren a los usuarios finales, además de comprender las interacciones entre cuidadores, pacientes y familiares ${ }^{(31)}$.

\section{DISCUSIÓN}

La revisión ayudo a describir la evidencia disponible sobre las IAAS y su asociación con el cuidador en adultos hospitalizados.

Si bien existen muchos factores de riesgo que pueden desencadenar una infección, los estudios, las estrategias y las políticas se han dirigido al lavado de manos, así mismo el personal de salud es el encargado de cumplir con las medidas de prevención, sin embargo, existe otro elemento importante en el momento de cuidado, el cuidador; que apoya en el cuidado y probable agente de infección.

Las políticas de aislamiento para los pacientes que desarrollen una IAAS genera seguridad, satisfacción y respuesta positiva de los cuidadores familiares, pero disminuye el tiempo de contacto y examen directo de los trabajadores de la salud lo que impacta en la calidad de la atención, además de que genera mayores eventos adversos, depresión y delirio del paciente, en este contexto se pone de relieve la generación y exploración de estudios que investiguen a fondo la atención que brindan, además de generar estrategias para mejorar el conocimiento de los cuidadores sobre la transmisión y la reducción a la exposición a los agentes causales ${ }^{(32) .}$

La revisión tuvo algunas limitaciones, primero, solo incluimos estudios publicados en 4 bases de datos, por lo tanto, los artículos relevantes publicados en otras bases de datos se podrían haber perdido, en segundo lugar, los metaanálisis evaluados surgieron de estudios descriptivos/ observacionales, que son susceptibles de sesgo. En tercer lugar hay muy pocos estudios que analicen la correlación entre el cuidador y las IAAS.

\section{CONCLUSIONES}

El gran porcentaje de estudios, estrategias y políticas se han dirigido al lavado de manos, así mismo el personal de salud es el encargado de cumplir con las medidas de prevención, sin embargo, existe otro elemento importante en el momento de cuidado, el cuidador que apoya en el cuidado y que no ha sido estudiado en el contexto de formar parte de la cadena de infección.

Las IAAS más comunes son las que predominan a nivel global en practicamente todas las instituciones de salud, algunas de ellas el cuidador puede ser un probable agente de infección.

Desafortunadamente los factores de riesgo son tan diversos, que los programas de prevención de IAAS, debe ser integrales e involucrar a directivos, administradores, personal de salud, instituciones educativas, personal de salud en formación, pacientes y cuidadores informales.

La tendencia indica que los cuidadores son personas con 
nivel educativo que posee las competencias de comprensión para ser participe de programas dirigidos a la prevención de las IAAS, se requiere rediseñar de la misma manera las estratégias educativas.

Se sugiere generar conciencia de la prevención tomando como base la educación, mejoramiento de las instalaciones hospitalarias, protocolos y procedimientos individualizados, generación de políticas institucionales y uso de la multimodalidad para los cuidadores y pacientes.

Se propone aumentar la producción científica que estudie la asociación que existe entre el cuidador y las IAAS en los hospitales, para evidenciar si existe una relación entre estas dos variables, planificar e implementar estrategias para la prevención, control y monitoreo, ubicando al cuidador, visitante y el propio paciente como actores fundamentales para la operacionalización de las mismas.

\section{CONFLICTO DE INTERESES}

Ninguno declarado

\section{FINANCIAMIENTO}

No existió financiamiento

\section{REFERENCIAS BIBLIOGRÁFICAS}

1. P.M. Olaechea PM. Insausti J. Blanco A. Luque P. Epidemiología e impacto de las infecciones nosocomiales. Medicina Intensiva 2010; 34 (4): 225-290. Disponible en: http://www.medintensiva.org/es-pdf-S0210569109001673.

2. OPS. Organización Panamericana de la Salud. Vigilancia epidemiológica de las infecciones asociadas a la atención en salud. Módulo III. Información para gerentes y personal directivo. Disponible en http://iris.paho.org/xmlui/bitstream/ handle/123456789/3270/OPS-Vigilancia-InfeccionesModulo-III-2012. pdf? sequence=1\&isAllowed $=y$

3. Fariñas AC. Teira CR. Rodríguez CP. Infección relacionada con la asistencia sanitaria (infección nosocomial). Medicine 2014; 11 (57): 3364-73. Disponible en: http://www.elsevier.es/ es-revista-medicine-62-articulo-infeccion-relacionada-conasistencia-sanitaria-S0304541214707851? referer=buscador.

4. Merzougui L. Barhoumi T. Guizana T. Barhoumi H. Hannachi $H$. Turki E. Et al. Les infections nosocomiales en milieu de réanimation: incidence annuelle et aspects cliniques au Service de Réanimation Polyvalente, Kairouan, Tunisie, 2014. Pan African Medical Journal 2018; 30:143. Disponible en: doi: 10.11604 / pamj.2018.30.143.13824.

5. Metsini A. Vázquez M. Sommerstein R. Marschall J. Voide C. Troillet N. Et al. Point prevalence of healthcare-associated infections and antibiotic use in three large Swiss acute-care hospitals. Swiss Med Wkly. 2018; 148: w14617. Disponible en: https://doi.org/10.4414/smw.2018.14617.

6. Unahualekhaka A. Epidemiología de las infecciones asociadas a la atención de salud. United Kingdom: International Federation of Infection Control 2015. Disponible en: http:// http://theific.org/wp-content/uploads/2014/08/ Spanish_ch3_PRESS.pdf

7. Kołpa M. Wałaszek M. Gniadek A. Zdzisław W. Wiesław D. Incidence, Microbiological Profile and Risk Factors of Healthcare-Associated Infections in Intensive Care Units: A 10 Year Observation in a Provincial Hospital in Southern Poland. En t. J. Environ. Res. Salud Pública 2018; 15 (1), 112. Disponible en doi: 10.3390 / ijerph15010112.

8. Llanos CA. Transmisión de infecciones nosocomiales por el personal de salud. Rev Med Hered 2016; 27 (2):7374. Disponible en: http://www.redalyc.org/articulo.oa?id= 338046643001.

9. Arefian H. Hagel S. Heublein S. Rissner F. Scherag A. Brunkhorst FM. Et al. Extra length of stay and costs because of health care-associated infections at a German university hospital. Rev American Journal of Infection Control 2016; 44 150-155. Disponible en: https://doi.org/10.1016/j. ajic.2015.09.005.

10. Sickbert BE. DiBiase LM. Schade WT. Wolak ES. Weber DJ. Rutala WA. Reducing health care-associated infections by implementing a novel all hands on deck approach for hand hygiene compliance. Rev American Journal of Infection Control. 2016; 44 13-16. Disponible en: https://doi. org/10.1016/j.ajic.2015.11.016

11. Maldonado LN. Luna VM. Lopera VV. Robledo J. Robledo RC. Characterization of the infection prevention and control programs in hospitals located in Medellín - Colombia, 2011. Rev Infectio. 2013; 17 (3): 136-145. Disponible en: https://doi. org/10.1016/S0123-9392(13)70720-3

12. Castañeda MF. Valdespino PM. Prevalencia de infecciones nosocomiales en un hospital de segundo nivel de atención en México. Rev Med IMSS 2015; 53 (6):686-690. Disponible en: http://www.redalyc.org/articulo.oa?id=457744940004.

13. Comisión Nacional de Arbitraje Médico. Frecuencia de infecciones asociadas a la atención de la salud en los principales sistemas de información de México. Boletín CONAMED-OPS. 2018; 3 (17). Disponible en http://www. conamed.gob.mx/gobmx/boletin/pdf/boletin17/frecuencia_ infecciones.pdf

14. Ambriz LM. Villalobos AL. Gallegos TR. Xeque MA. Hernández CM. Palomé VG. Perfil del cuidador principal y su relación con el cuidado del personal de enfermería. Rev Enferm Inst Mex Seguro Soc 2014; 22 (3): 123-9. Disponible en: http://www.medigraphic.com/pdfs/enfermeriaimss/eim- 
2014/eim143b.pdf.

15. Flores ME, Fuentes HL, González GJ, Meza IJ, Cervantes GA, Valle MA. Perfil del cuidador primario informal del adulto mayor hospitalizado: Academia Journals 2016; Disponible en: https:// file:///C:/Users/COMUNIDAD/Desktop/ ARTICULOPUEBLA_2016MaElena.pdf.

16. Villar DR. Pancorbo HP. Jiménez DM. Cruz LA. García RP. Qué hace el cuidador familiar en el hospital. Cómo se ve a sí mismo y cómo lo ven los profesionales. Gerokomos 2012; 23 (1): 7-14. Disponible en: http://scielo.isciii.es/pdf/geroko/ v23n1/comunicacion1.pdf.

17. Quiñoz GM. Vellido GC. Rivas CA. Martín BM. González GL. Vellido GD. Et al. Evaluación del plan funcional dirigido a personas cuidadoras en hospitalización. Rev Enferm Clin 2013; 23 (6): 262-270. Disponible en: http://dx.doi. org/10.1016/j.enfcli.2013.08.008.

18. Rizo A, Molina L, Milián N, Pagán P. Machado J. Caracterización del cuidador primario de enfermo oncológico en estado avanzado. Rev Cubana Med Gen Integr 2016; 32 (3). Disponible en: http://scielo.sld.cu/scielo.php?script=sci_ arttext\&pid=S0864-21252016000300005\&lng=es.

19. INEGI. Instituto Nacional de Estadística y Geografía: Estadísticas a propósito del día de las y los cuidadores de personas dependientes. Disponible en: http://www.inegi. org.mx/saladeprensa/aproposito/2017/cuidadores2017_Nal. pdf.

20. Real Academia Española (internet). Madrid 2018. Disponible en: https://www.rae.es.

21. Ahmed KH. Kanwal BF. Mehboob R. Nosocomial infections: Epidemiology, prevention, control and surveillance. Asian Pacific Journal of Tropical Biomedicine 2017; 7 (5) 478-482. Disponible en: https://doi.org/10.1016/j.apjtb.2017.01.019.

22. Rodríguez-Aceles AL. Abreu AM. Engelman B. CañónMontañez W. Risk factors for health care-associated infection in hospitalized adults: Systematic review and meta-analysis. American Journal of Infection Control 2017; 45 (12) 149-156. Disponible en: https://doi.org/10.1016/j.ajic.2017.08.016.

23. Conway L. Carter E. Larson E. Risk Factors for Nosocomial Bacteremia Secondary to Urinary Catheter-Associated Bacteriuria: A Systematic Review. Urol Nurs 2015; 35 (4): 191-203. Disponible en: https://www.ncbi.nlm.nih.gov/pmc/ articles/PMC4586038/.

24. Yahav D. Eliakim-Raz N. Leibovici L. Paul M. Bloodstream infections in older patients. Journal Virulence 2016; 7 (3): 341-352. Disponible en: https://doi.org/10.1080/21505594.2 015.1132142.
25. Bono V. Giacobbe D. Bloodstream infections in internal medicine. Journal Virulence 2016; 7 (3): 353-365. Disponible en: https://doi.org/10.1080/21505594.2016.1140296.

26. Cuartas-Castro VM. Cano-Velásquez AM. Factores psicosociales en cuidadores y riesgo de infección del torrente circulatorio en pacientes en una institución de Medellín, 2014. CES Salud Pública 2015; 6: 12-20. Disponible en: revistas.ces.edu.co/index.php/ces_salud_publica/article/ download/2976/2413.

27. Horng LM. Unicomb L. Alam M. Halder AK. Shoab AK. Ghosh PK. Et al. Healthcare worker and family caregiver hand hygiene in Bangladeshi healthcare facilities: results from the Bangladesh National Hygiene Baseline Survey. The Journal of Hospital Infection 2016; 94 (3): 286-294. Disponible en: https://doi.org/10.1016/j.jhin.2016.08.016.

28. Chittick P. Koppisetty S. Lombardo L. Vadhavana A. Solanki A. Cumming K. Et. Al. Assessing patient and caregiver understanding of and satisfaction with the use of contact isolation. Revista Americana de Control de Infecciones 2016; 44 (6): 657-660. Disponible en: https://doi.org/10.1016/j. ajic.2015.12.033.

29. Roidad N. Khakoo R. Knowledge and attitudes of visitors to patients in contact isolation. Revista Americana de Control de Infecciones 2014; 42: 198-199. Disponible en: https://doi. org/10.1016/j.ajic.2013.09.004.

30. Luby P. Sultana R. Ali RN. Zaman R. Uddin P. Nahar N. Et al. Family caregivers in public tertiary care hospitals in Bangladesh: Risks and opportunities for infection control. Revista Americana de Control de Infecciones 2014; 42 (3): 305-310. Disponible en: https://doi.org/10.1016/j. ajic.2013.09.012.

31. Jefrey NJ. Sagha-Zadeth MR. Vielemeyer O. Franklin E. Evidence-based practices to increase hand hygiene compliance in health care facilities: An integrated review. American Journal of Infection Control 2017; 44 (6): 691-794. Disponible en: https://doi.org/10.1016/j.ajic.2015.11.034.

32. Seibert G. Ewers T. Barber A. Slavick A. Wright MO. Stevens $L$. Et al. What do visitors know and how do they feel about contact precautions. Revista Americana de Control de Infecciones 2018; 46 (1): 115-117. Disponible en: https://doi. org/10.1016/j.ajic.2017.05.011.

33. Islam Saiful; Luby Stephen; Sultana Rebeca; Uz Rashid; Uddin Principal; Nahar Nazmun; et al. Family caregivers in public tertiary care hospitals in Bangladesh: Risks and opportunities for infection control. American Journal of Infection Control 2014; 42 (3) 305-310. Disponible en: https:// doi.org/10.1016/j.ajic.2013.09.012. 